\section{Vibration Isolation Critical to Measuring Neuronal Patterns in the Brain}

\author{
David L. Platus \\ Minus K Technology, Inc., Inglewood, California \\ david@minusk.com
}

Researchers at Georgetown University's Department of Physiology and Biophysics use negative-stiffness vibration isolators to help measure micron-level patterns of neuronal activity in the mammalian neocortex. The research is shedding new light into brain sensory and motor processing functions relating to cardiac fibrillation and epilepsy.

Isolating a laboratory's sensitive microscopy equipment against low-frequency vibration has become increasingly more vital to maintaining imaging quality and data integrity for neurobiology researches. Ever more frequently, laboratory researchers are discovering that conventional air tables and the more recent active (electronic) vibration isolation systems are not able to adequately cancel out the lower frequency perturbations derived from air conditioning systems, outside vehicular movements and ambulatory personnel. Such was the case with the Department of Physiology and Biophysics at Georgetown University Medical Center, where Professor Jian-Young Wu has been conducting research on waves of neuronal activity in the neocortex of the brain.

\section{Propagating Waves in Neocortical Slices}

$\mathrm{Wu}$ and his colleagues visualize wave-like patterns in the brain cortex using a new method called voltage-sensitive dye imaging. They use a special dye that binds to the membrane of neurons and changes color when electrical potential changes on the membrane of active neurons. The neuronal sample is derived from slices of rat neocortex.

The neocortex is the outer layer of the cerebral hemispheres in the brain of mammals. Made up of six layers, it is involved in higher functions such as sensory perception, generation of motor commands and, in humans, language. The neurons of the neocortex are ar-

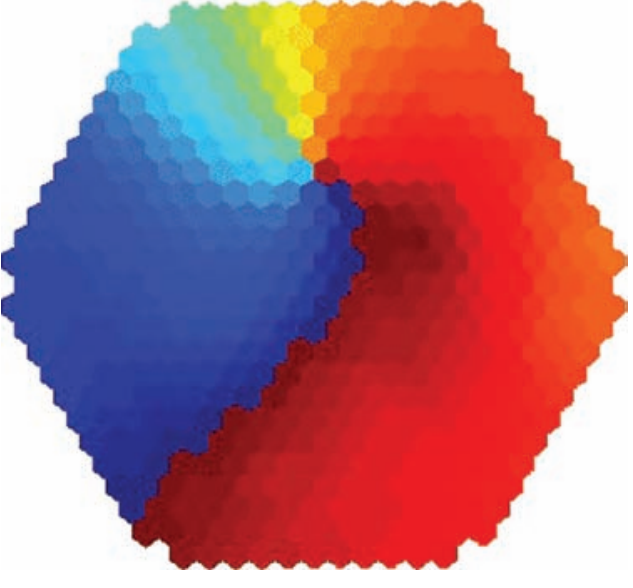

Image of neuronal patterns in the rat neocortex - Georgetown University. ranged in vertical

structures called neocortical columns. These are patches of the neocortex with a diameter of about $0.5 \mathrm{~mm}$ and a depth of $2 \mathrm{~mm}$. Each column typically responds to a sensory stimulus representing a certain body part, or region of sound or vision. In the human neocortex, it is postulated that there are about a half-million of these columns, each of which contains approximately 60,000 neurons.
The neocortex can be viewed as a huge web, consisting of billions to trillions of neurons and hundred of trillions of interconnections. While individual neurons are too simple to have intelligence, the collective behavior of the billions of interneuronal interactions occurring each second can be highly intelligent.

Traditionally, scientists have studied brain activity by placing a few electrodes in the brain and measuring the electrical signals of the neurons close to the electrodes. This method is workable for understanding the function of the cortex and interactions between individual neurons, but it is not suitable for studying the emerging properties of the nervous system. It is like viewing a few pixels on a television screen and trying to figure out the story. Now, with optical methods and voltage-sensitive dyes, Georgetown University researchers can visualize the activation in a large area of the neocortex when the brain is processing sensory information, similar to watching the whole television screen.

Voltage-sensitive dye is a compound that stains neuronal membrane and changes its color when the neuron is excited. This allows $\mathrm{Wu}$ to visualize population neuronal activity dynamically in the cortex and study how individual neurons in the neocortex interact to generate population neuronal activities that underlie sensory and motor processing functions. Population activities are composed of the coordinated activity of up to billions of neurons. Currently, his team studies how oscillations and propagating waves can be generated by small ensembles of neocortical neurons.

Viewing the spatiotemporal patterns of neuronal population in the cortex is markedly different from recording individual neurons. Here the cortical activity is viewed as "population activity," that can be more complex than the linear addition of an individual neuron's activity. Voltage-sensitive dye and optical recording techniques give the neuroscientist a new tool for figuring out how the brain cortex works.

\section{Spiraling Waves in the Brain Shed Light on Cardiac Fibrillation and Epilepsy}

The Georgetown imaging team has uncovered spiraling wave patterns resembling little hurricanes in the brain. He believes that this hurricane-like spiral pattern is an emergent behavior of the network. Just as a metrological hurricane is an emergent behavior of a large volume of air molecules, if you were to dissect a hurricane into individual air molecules you would not find any special process that generates a hurricane. Similarly, the Georgetown team has found that in the nervous system, spiral waves are an emergent process of the neuronal population and there might be no special cellular process attributed to spirals. But like a hurricane, spiral waves can be a powerful force for organizing the activity of a neuronal population. Spirals generated in a small area can send out a powerful storm that invades large, normal brain areas and starts a seizure attack. This hypothesis would mean that epilepsy could be viewed not just as a miswiring in the brain, but as an abnormal wave pattern that invades normal tissue.

Likewise, during cardiac fibrillation, spiral waves form in the heart emitting rotating and scroll waves in two and three dimensions. As a leading life-threatening situation, these rotating waves can kill the patient instantly as the pumping function 


\section{When you need vibration isolation you need Negative-Stifiness technology from Minus $K^{\odot}$}

\section{The best performance and the lowest price. Now that's hard to beat!}

\section{Why have over 2,000 scientists in 35 countries selected Minus K${ }^{\circledR}$ vibration isolators?}

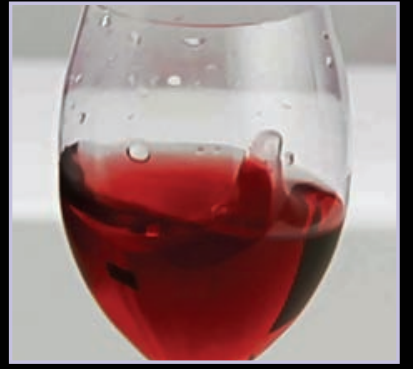

Without Minus $\mathbf{K}^{\circledR}$

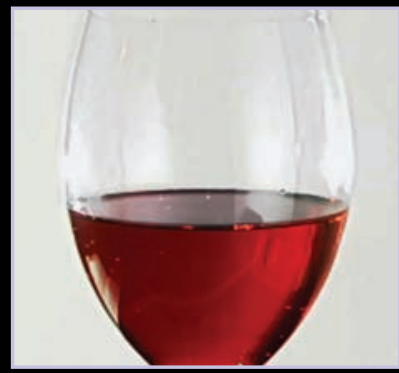

With Minus $\mathbf{K}^{\circledR}$

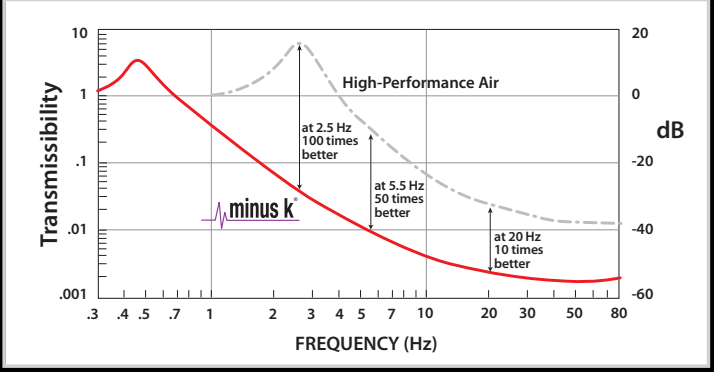

Our Transmissibility curve speaks for itself.

Minus $\mathrm{K}^{\circledR}$ vibration isolation systems deliver 10x to $100 \mathrm{x}$ better performance than high-performance air systems and even better than aotive systems.

\section{Improve your images with patented Negative-Stiffness technology.}

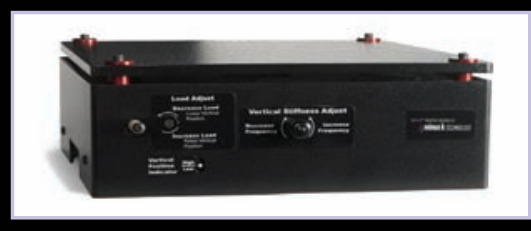

Bench Tops

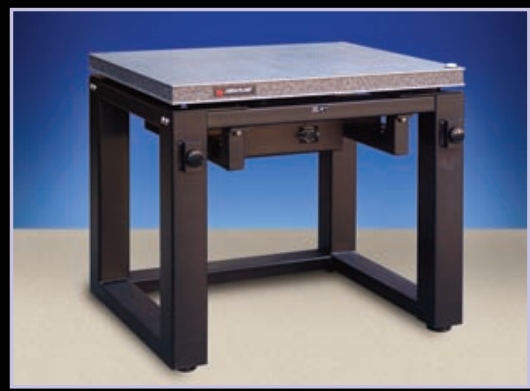

Workstations

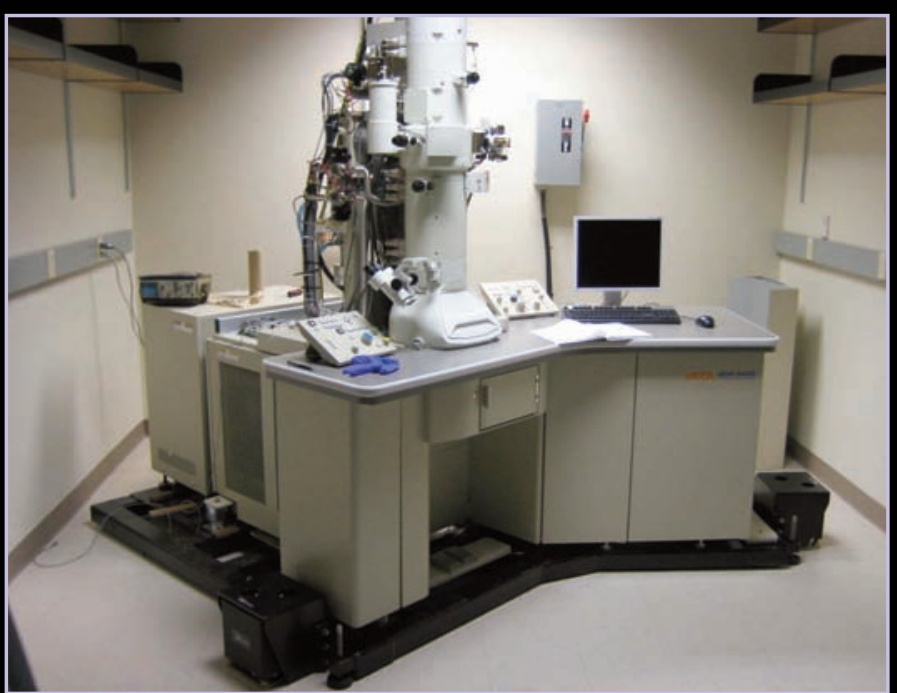

Floor Platforms
Visit www.minusk.com for more information...

VIBRATION ISOLATION BY:

\section{minUS $k^{\circ}$ TECHNOLOGY}

460 S. Hindry Ave., Unit C, Inglewood, CA 90301

Tel: 310-348-9656 Fax: 310-348-9638

sales@minusk.com • www.minusk.com
Custom Applications -

Give us your Vibration Isolation Challenge

As featured in Laser Focus World, Motion System Design, Machine Design, Design News, Photonics Spectra, Laboratory Equipment, The Scientist, R \& D Magazine and Microscopy Today. 
of the heart is disrupted by the 5 to $10 \mathrm{~Hz}$ rotations that drives chaotic and abnormally rapid cardiac contractions.

Wu believes that propagating waves are a basic pattern of cortical neuronal activity, and that these wave patterns may play an important role in initiating and organizing brain activity involving millions to billions of neurons. Studying the spatiotemporal patterns of neuronal population activity may provide insight into normal brain functions and pathological disorders. This research has the potential to help scientists understand abnormal waves that are generated in the brains of patients with epilepsy.

\section{Vibration Isolation}

Since voltage-sensitive dye signals are small, usually a change of 0.1 to 1.0 percent of the illumination intensity, the Georgetown team has used a high-dynamic-range camera, photodiode array to detect the voltage-sensitive dye signals of the cortical activity. The photodiode array can resolve extremely small changes in light, usually one part of ten thousands. (Human eyes and ordinary digital cameras register light changes of one part to a hundred). Detecting such small signals requires an extreme isolation of vibration. The lab had to contend with low frequency vibrations from air conditioning equipment, people walking, and wind blowing against the building. Vibrations as low as $1 \mathrm{~Hz}$ were inhibiting the integrity of the images and data.

At first, high-quality air tables were utilized, but they were not adequate for isolating low frequency vibrations. They then tried putting a second air table on top of the first one, but that still did not give them the isolation needed. Then they tried an active, electronic system, but were still spending much time fighting with floor vibrations. Effectively, the lab was dealing with an unresolved vibration problem for many years.

The Georgetown lab eventually tested, and settled upon

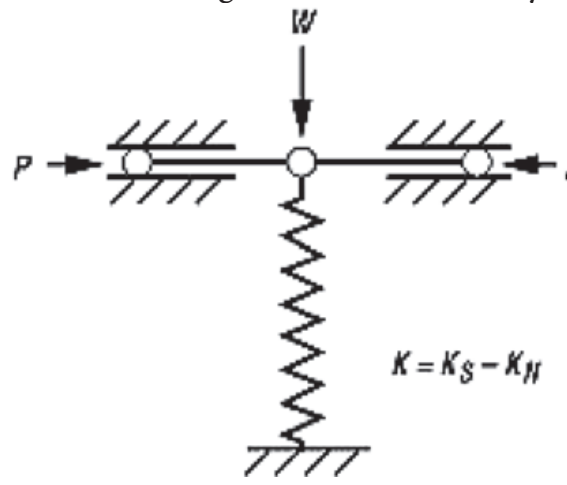

Figure 1 A vertical-motion isolator, as demonstrated in figure 1 , uses a conventional spring connected to an NSM consisting of two bars hinged at the center, supported at their outer ends on pivots, and loaded in compression by forces $P$. (The hinged bars are for illustration only. Flexures are used in the isolators to avoid stiction and friction.) The spring is compressed by weight $W$ to the operating position of the isolator. The stiffness of the isolator is $K=K_{S}-K_{N}$ where $K_{S}$ is the spring stiffness and $K_{N}$ is the magnitude of a negative stiffness which is a function of the length of the bars and the load $P$. The isolator stiffness can be made to approach zero while the spring supports the weight $W$.

negative-stiffness mechanism vibration isolation systems from Minus K Technology, which enabled the lab to get vibration isolation down to a level of $1 \mathrm{~Hz}$. This effectively cancelled out any vibration noise difficulties that were inhibiting image and data readings. [1.-4.]

Negative-stiffness mechanism (NSM) isolators have the flexibility of custom tailoring resonant frequencies vertically and horizontally. They employ a completely mechanical concept in low-frequency vibration isolation. Vertical-
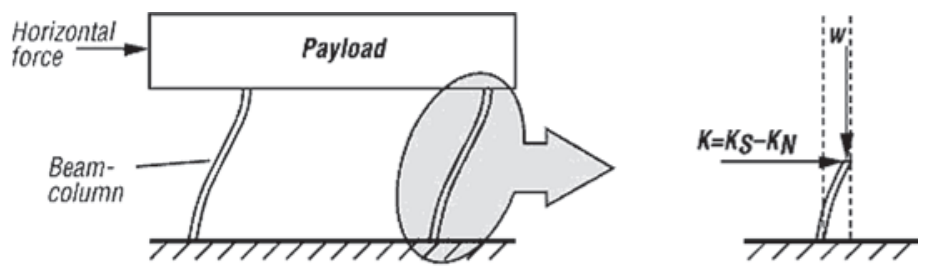

Figure 2 A horizontal-motion isolation system, as demonstrated in figure 2, is illustrated by two beam-column isolators. Each isolator behaves like two fixed-free beam-columns loaded axially by a weight load $W$. Without the weight load the beam-columns have horizontal stiffness $K_{S}$. With the weight load the lateral bending stiffness is reduced by the "beam-column" effect. This behavior is equivalent to a horizontal spring combined with an NSM so that the horizontal stiffness is $K=K_{S}-K_{N}$, and $K_{N}$ is the magnitude of the beam-column effect. Horizontal stiffness can be made to approach zero by loading the beam-columns to approach their critical buckling load.

\section{Schematic of Negative-Stiffness Isolator}

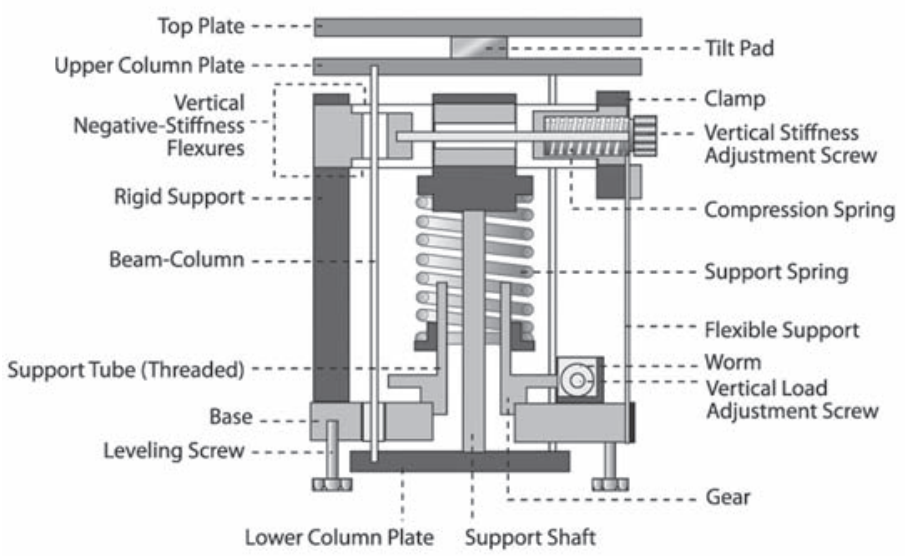

Figure 3 Schematic of a NSM vibration isolator, as shown in figure 3. A vertical stiffness adjustment screw is used to regulate the compression force on the negative-stiffness flexures. A vertical load adjustment screw raises or lowers the base of the support spring in response to varying weight loads to keep the flexures in their straight, unbent operating position. motion isolation is provided by a stiff spring that supports a weight load, combined with a NSM. The net vertical stiffness is made very low without affecting the static load-supporting capability of the spring.

Beam-columns connected in series with the vertical-motion isolator provide horizontal-motion isolation. The horizontal stiffness of the beam-columns is reduced by the "beam-column" effect. A beam-column behaves as a spring combined with a

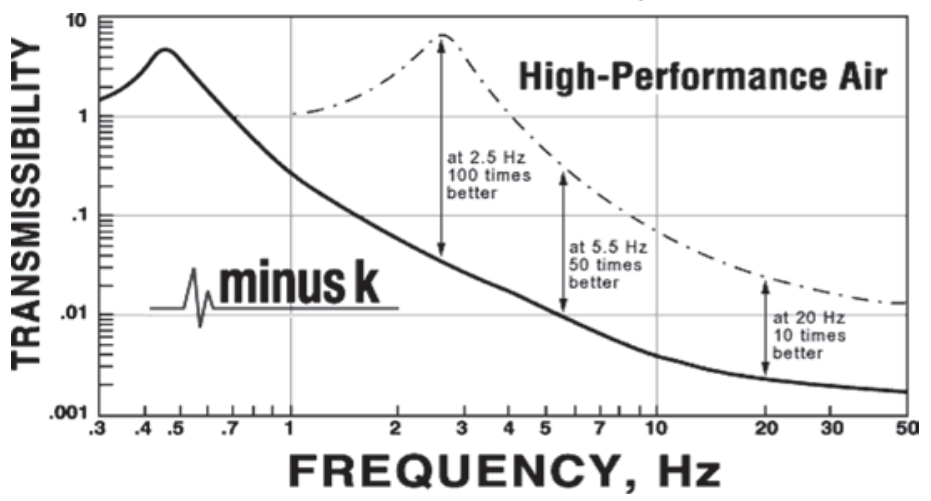

The transmissibility of a passive negative-stiffness vibration isolator, as shown in figure 4, the vibration that transmits through the isolator as measured as a function of input vibrations - can be 10X to $100 \mathrm{X}$ better than high-performance air tables depending on the vibration frequency. 
NSM. The result is a compact passive isolator capable of very low vertical and horizontal natural frequencies and very high internal structural frequencies.

Transmissibility with negative-stiffness is substantially improved over air systems, which can make vibration isolation problems worse since they have a resonant frequency that can match that of floor vibrations. Transmissibility is a measure of the vibrations that transmit through the isolator relative to the input vibrations. The NSM isolators, when adjusted to $0.5 \mathrm{~Hz}$, achieve 93 percent isolation efficiency at $2 \mathrm{~Hz}$; 99 percent at $5 \mathrm{~Hz}$; and 99.7 percent at $10 \mathrm{~Hz}$.

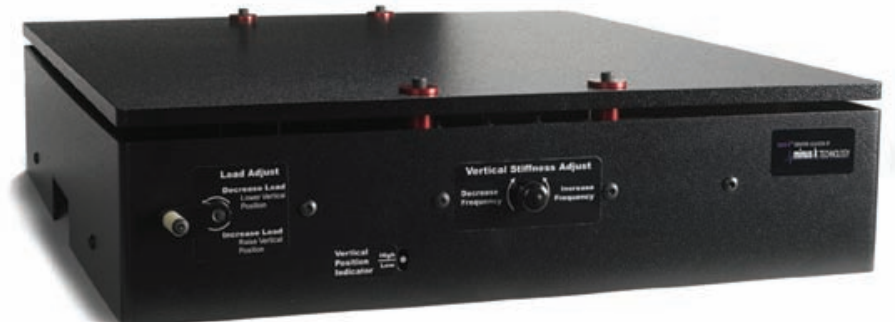

The Minus K BM-8 bench-top vibration isolation platform is lowprofile at 4.6 inches tall, does not require air or electricity, and is portable and user-friendly.

NSM transmissibility is also improved over active systems. Because they run on electricity, active systems can be negatively influenced by problems of electronic dysfunction and power modulations, which can interrupt scanning. They also have a limited dynamic range - which is easy to exceed - causing the isolator to go into positive feedback and generate noise under- neath the equipment. Although active isolation systems have fundamentally no resonance, their transmissibility does not roll off as fast as negative-stiffness isolators.

\section{Continuing Research}

Within the past ten years, Dr.Wu's team has documented a variety of waveforms (e.g., plane wave and spirals) in brain slices during artificially induced oscillations. Using neocortical slices and mathematical models, they are studying the initiation of the waves and the factors that control their propagating direction and velocity.

The lab is also involved in the development of new optical imaging techniques - relying on the negative-stiffness vibration isolation systems - such as imaging propagating waves in vivo, in intact brains, which is technically difficult because other imaging methods (MRI, PET or MEG) provide inadequate spatiotemporal resolution. Large-scale neuronal activity is a hallmark of a living brain. Wu's team is improving the optical imaging techniques with a hope to visualize the waves in the cortex in vivo during sensory processes and in awake animals while behavioral and cognitive tasks are performed.

\section{References:}

1. David K. Ferry, Ph.D., Negative-Stiffness Vibration Isolation Improves Reliability of Nanoinstrumentation, Laser Focus World, October, 2007.

2. L.B. Cohen, More Than Just Floating on Air, BioPhototonics. International, March 2008.

3. Jim McMahon, Vibration Isolation in Cleanrooms, Controlled Environments, January 2008.

4. P. O'Hara, Mechanical Isolators Stop the Shakes, Design News, March 2007.
Fast, Low Temperature Microwave Tissue Processing

- Rapid Specimen Turnaround

- Low Temperature Environment

- Programmable Processing

- Consistent Quality Results

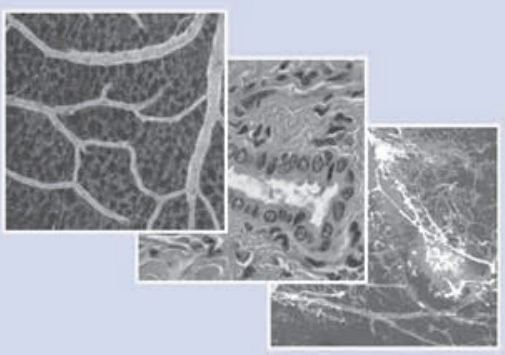

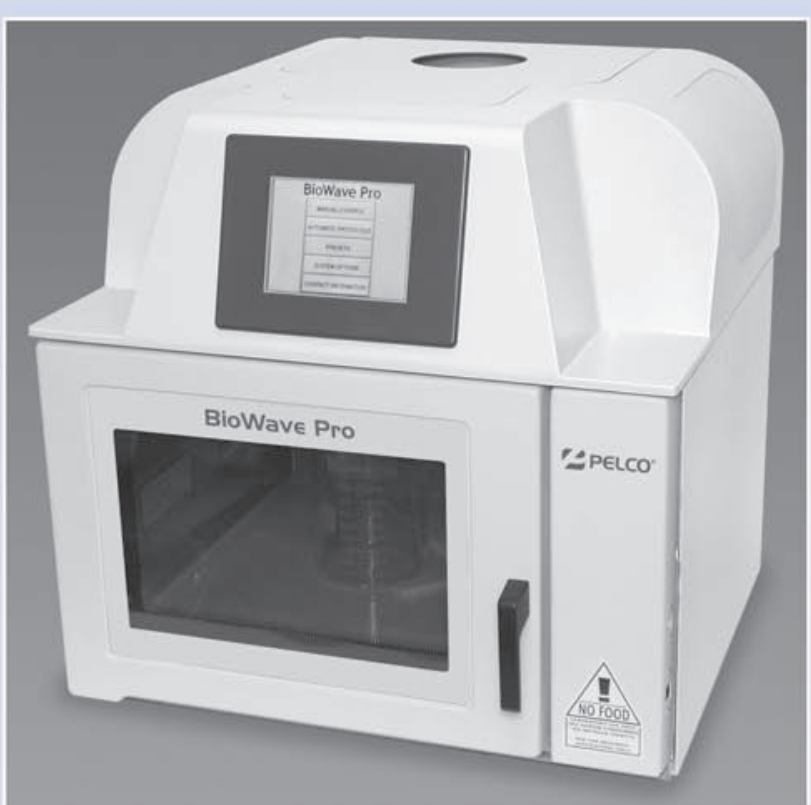

Application kits for paraffin processing, decalcification, confocal, light and electron microscopy are available.

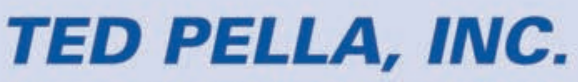

Microscopy Products for Science and Industry

sales@tedpella.com・Tel: 800-237-3526•www.tedpella.com 